\title{
Conditional Currency Hedging
}

\author{
Melk C. Bucher
}

Angelo Ranaldo

Swiss Institute of Banking and Finance, University of St.Gallen

melk.bucher@unisg.ch

Preliminary work. Comments welcome

EFMA Basel 07/02/2016 


\section{Research Question and Overview of Problem}

\section{How to optimally hedge currency risk?}

- Global investors inherently face currency (FX) exposure through their international investments

- Question of optimal hedging of FX risk is thus inevitable

- Literature: No hedging, full hedging, unconditional mean-variance hedging ... how about conditional hedging?

- What is the target of FX hedging? (FX risk, total portfolio risk, risk/return) 


\section{Agenda}

(1) Contribution to literature \& Motivation

(2) Research design

(3) Major results

(4) Conclusion 


\section{Outline}

(1) Contribution to literature \& Motivation

(3) Major results 


\section{Optimal FX exposure in the literature}

- Speculative demands

(1) Siegel paradox (Siegel, 1972).

(2) Expected excess returns, such as the carry trade (Hansen \& Hodrick, 1980; Fama, 1984; Hodrick, 1987; Engel, 1996; Burnside et al., 2006; Brunnermeier, Nagel \& Pedersen, 2009)

- Risk management (Hedging):

(1) No domestic asset riskless in real terms (Adler and Dumas, 1983).

(2) Risk management demands depend on correlation with given baseline assets.

(3) If equities and currencies are uncorrelated, risk management demands are zero, implying full FX hedging (Solnik, 1974).

(4) Optimal (unconditional) mean-variance hedging strategies can reduce total return volatility (Campbell, Serfaty-de-Medeiros \& Viceira, 2010; Glen \& Jorion, 1993).

\section{Research gap}

What if time-varying correlation structure is conditional on certain risk factors? Question of Conditional Currency Hedging. 


\section{Conditioning information: global risk factors}

- What can serve as useful information to predict future inter-asset class correlation? Global and FX risk factors with forward-looking content

- Recent literature on global risk factors across asset classes (e.g. Lettau, Maggiori \& Weber, 2014; Cenedese, Payne, Sarno \& Valente, 2016)

- implied equity and FX volatility; downstate pricing (DR-CAPM)

- Recent literature in FX asset pricing: risk factors accounting for the Carry Trade and Momentum excess returns (e.g. Lustig et al., 2011; Burnside, 2012; Menkhoff et al., 2012a; Mancini et al., 2013; Mueller et al., 2012; Ready et al., 2015; Richmond, 2016)

- DOL, HML, VOL, MOM, IML, COR, IMX, PMC

\section{Research gap}

Use lagged FX and global risk factors to predict future across-asset correlation and thus conditional hedging 


\section{An empirical motivation for conditional currency hedging}

Figure 1: Correlation of global equities, bonds and commodities with $D O L_{F X}$ and $H M L_{F X}$ across lagged implied FX volatility (1995:02-2015:12)
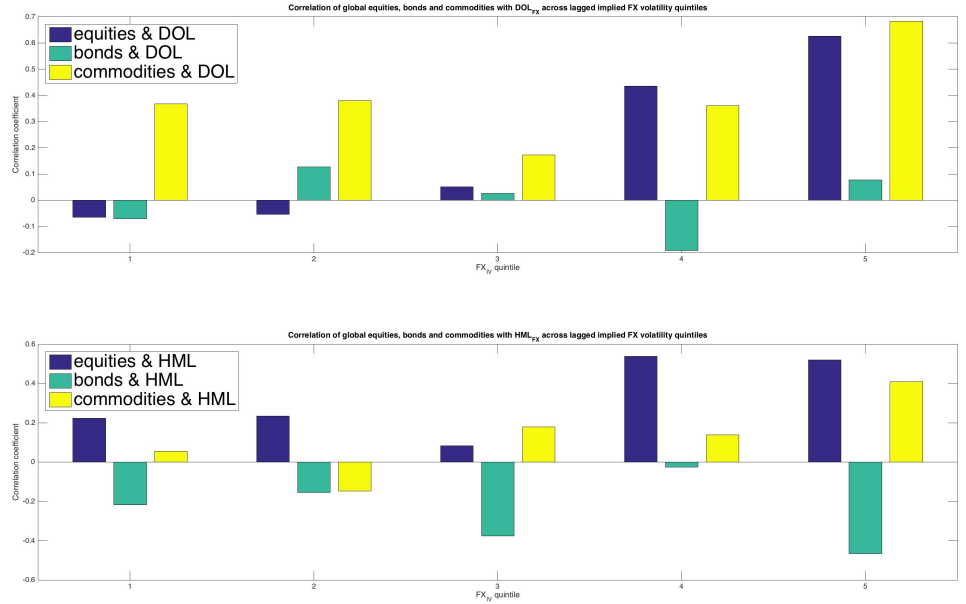


\section{An empirical motivation for conditional currency hedging}

Figure 2: Correlation of global equities with six G20 currency pairs (against USD) across lagged implied FX volatility (1995:02-2015:12)

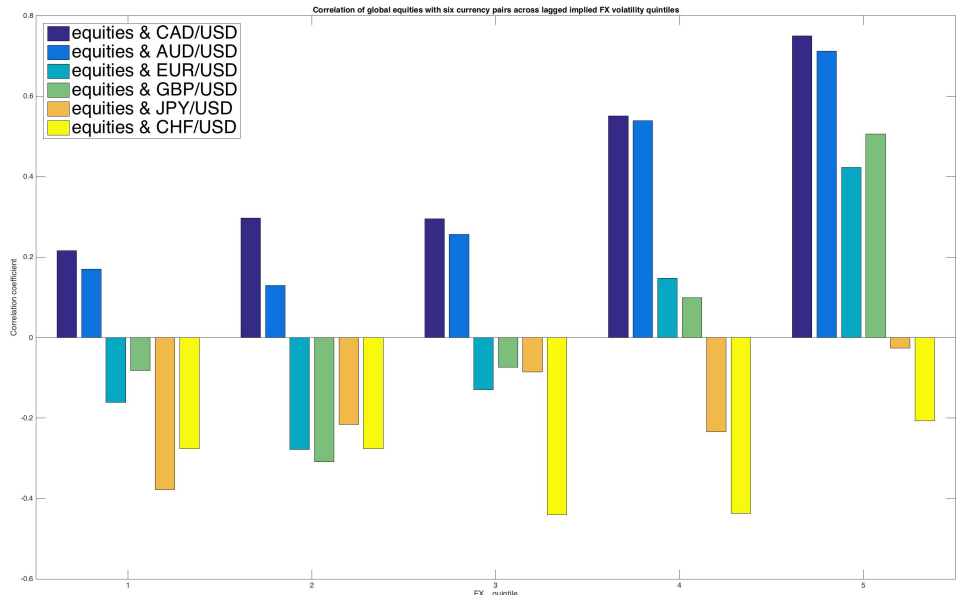




\section{Outline}

\section{(1) Contribution to literature \& Motivation}

(2) Research design

(3) Major results

(4) Conclusion 


\section{Data}

- 7 developed countries: Australia, Canada, Eurozone, Japan, Switzerland, UK, USA (data going back the longest)

- Data sources:

(1) Stocks: $\mathrm{MSCl}$ country indices

(2) Bonds: 10 Year Yields, $3 \mathrm{~m}$ interest rates, FX against USD from International Financial Statistics database (IMF); if not available, OECD

(3) Global risk factors: VIX pulled from Wharton Research Database; FX implied volatility from Datastream (1995:2-2015:12); FX risk factors from authors (Menkhoff et al., 2012b; Mueller et al., 2012; Ready et al., 2015; Richmond, 2016)

- Data transformation: Excess log returns over domestic risk-free rate $(3 \mathrm{~m})$; Log bond returns using approximation suggested in Campbell, Lo \& MacKinlay (1997)

- Baseline analysis in monthly returns; Robustness with $3 \mathrm{~m}$ (non)overlapping returns 


\section{Methodology}

\section{Mean-variance OLS approach to minimize total portfolio variance}

$$
\begin{gathered}
r_{p, t+1}^{h}-i_{1, t}=\mathbf{1}^{\prime} \boldsymbol{\omega}_{\mathbf{t}}\left(\boldsymbol{r}_{\mathbf{t}+\mathbf{1}}-\boldsymbol{i}_{\mathbf{t}}\right)+\boldsymbol{\Psi}_{\mathbf{t}}^{\prime}\left(\boldsymbol{\Delta} \mathbf{s}_{\mathbf{t}+\mathbf{1}}+\mathbf{i}_{\mathbf{t}}-\mathbf{i}_{\mathbf{t}}^{\mathbf{d}}\right)+\frac{1}{2} \sum_{t}^{h} \\
\arg \min \left(\sigma_{p, t+1}^{2}\right) \\
\boldsymbol{\Psi}_{\mathbf{t}} \\
\mathbf{1}^{\prime} \boldsymbol{\omega}_{t}\left(\boldsymbol{r}_{\boldsymbol{t}+\mathbf{1}}-\boldsymbol{i}_{\boldsymbol{t}}\right)=\gamma_{0}-\boldsymbol{\Psi}_{\mathbf{u}}^{\prime}\left(\boldsymbol{\Delta} \mathbf{s}_{\mathbf{t}+\mathbf{1}}+\mathbf{i}_{\mathbf{t}}-\mathbf{i}_{\mathbf{t}}^{\mathbf{d}}\right) \\
-\boldsymbol{\Psi}_{\mathbf{c}, \mathbf{t}}^{\prime}\left(\Delta s_{t+1}+\boldsymbol{i}_{\boldsymbol{t}}-\boldsymbol{i}_{\mathbf{t}}^{\boldsymbol{d}}\right) R F_{t} \\
\boldsymbol{\Psi}_{\mathbf{t}}=\boldsymbol{\Psi}_{\mathbf{u}}+\boldsymbol{\Psi}_{\mathbf{c}, \mathbf{t}} R F_{t}
\end{gathered}
$$




\section{Methodology}

- Mean-variance approach: Variance minimization of total portfolio returns through optimal FX weights. Optimal FX weights arrived at by regression of domestic excess portfolio returns on FX excess returns and taking negative of regression coefficients (e.g. Campbell et al., 2010).

(1) Ordinary least squares (OLS) (Campbell, 2010). Optimal FX weights linear function of risk factor

(2) Logistic smooth transition regression model (LSTAR) (Christiansen, Ranaldo, Soderlind, 2011). Optimal FX weights logistic function of risk factor

- In- and out-of-sample testing of the models in terms of optimal FX weights as well as portfolio variance

- Primary: risk minimization; Secondary: Risk/return trade-off 


\section{Outline}

\section{(1) Contribution to literature \& Motivation}

(2) Research design

(3) Major results 


\section{Risk-minimizing conditional currency weights}

Table 1: Optimal Currency Exposure for the Equally-Weighted Global Equity Portfolio: Unconditional Case and Sensitivity to lagged implied and realized volatility

\begin{tabular}{|c|c|c|c|}
\hline & Unconditional & implied VOL & realized VOL \\
\hline & Estimate (Std. Error) & Estimate (Std. Error) & \\
\hline EUR_USD & $0.03(0.12)$ & $-1.47^{* *}(0.55)$ & $0.03(0.32)$ \\
\hline AUD_USD & $-0.25 * *(0.06)$ & $0.38(0.38)$ & $-0.16(0.20)$ \\
\hline CAD_USD & $-0.63 * * *(0.10)$ & $-0.35(0.39)$ & $-0.21(0.30)$ \\
\hline JPY_USD & $0.03(0.06)$ & $0.63 * *(0.23)$ & $0.27 *(0.15)$ \\
\hline CHF_USD & $0.27^{* *}(0.10)$ & $1.21 * *(0.37)$ & $0.30(0.25)$ \\
\hline GBP_USD & $-0.05(0.07)$ & $0.60 *(0.32)$ & $0.19(0.18)$ \\
\hline USD & $0.66^{* * *}(0.24)$ & NA & NA \\
\hline EUR $* V O L_{i}$ & & $0.06(0.05)$ & \\
\hline AUD $* V O L_{i}$ & & $-0.08 *(0.04)$ & \\
\hline $\mathrm{CAD} * V O L_{i}$ & & $-0.09 *(0.04)$ & \\
\hline$J P Y * V O L_{i}$ & & $-0.03(0.02)$ & \\
\hline $\mathrm{CHF} * V O L_{i}$ & & $0.35 *(0.17)$ & \\
\hline $\mathrm{GBP} * V O L_{i}$ & & $-0.04(0.03)$ & \\
\hline EUR $* V O L_{r}$ & & & $-0.06(0.69)$ \\
\hline AUD $* V O L_{r}$ & & & $-0.23(0.44)$ \\
\hline$C A D * V O L_{r}$ & & & $-0.81(0.64)$ \\
\hline$J P Y * V O L_{r}$ & & & $-0.58 *(0.31)$ \\
\hline $\mathrm{CHF} * V O L_{r}$ & & & $-0.02(0.51)$ \\
\hline $\mathrm{GBP} * V O L_{r}$ & & 4 & $-0.51(0.36)$ \\
\hline
\end{tabular}




\section{In-sample performance of various hedging regimes}

Table 2: Risk reduction due to conditional currency hedging

\begin{tabular}{rrrr}
\hline & Unconditional hedging (1976:3-2015:12) & implied VOL (1995:2-2015:12) & realized VOL (1976:3-2015:12) \\
& {$[$ Full hedging] } & {$[$ Full hedging] } & [Full hedging] \\
\hline \hline annualized return & $0.036[0.047]$ & $0.018[0.049]$ & $0.036[0.047]$ \\
annualized std & $0.119[0.134]$ & $0.09[0.135]$ & $0.117[0.134]$ \\
Sharpe ratio & $0.30[0.35]$ & $0.18[0.3]$ & $0.31[0.35]$ \\
Max drawdown & $-0.255[-0.286]$ & $-0.086[-0.158]$ & $-0.249[-0.286]$ \\
Skewness & $-1.419[-1.658]$ & $-0.539[-1.132]$ & $-1.45[-1.658]$ \\
Kurtosis & $9.88[10.62]$ & $3.28[4.97]$ & $9.95[10.62]$ \\
\hline
\end{tabular}

- In-sample, both unconditional as well as conditional hedging lead to a reduction in risk at the cost of lower expected returns

- Hedging through implied volatility strongly further decreases risk in terms of std. dev., drawdown, skewness and kurtosis relative to full and unconditional hedging. 


\section{Out-of-sample performance of various hedging regimes}

Table 3: Risk reduction due to conditional currency hedging

\begin{tabular}{rrrr}
\hline & UC optimal hedging $(1985: 1-2015: 12)$ & $V O L_{i}(1995: 2-2015: 12)$ & $V O L_{r}(1985: 1-2015: 12)$ \\
& {$[$ Full hedging] } & {$[$ Full hedging] } & [Full hedging] \\
\hline \hline annualized return & $0.023[0.049]$ & $0.056[0.065]$ & $0.049[0.049]$ \\
annualized std & $0.127[0.139]$ & $0.101[0.128]$ & $0.139[0.139]$ \\
Sharpe ratio & $0.18[0.35]$ & $0.55[0.51]$ & $0.36[0.35]$ \\
Max drawdown & $-0.283[-0.286]$ & $-0.085[-0.158]$ & $-0.302[-0.286]$ \\
Skewness & $-1.896[-1.750]$ & $-0.010[-1.264]$ & $-1.820[-1.750]$ \\
Kurtosis & $13.50[11.01]$ & $4.39[5.83]$ & $12.912[11.01]$ \\
\hline
\end{tabular}

- Out of sample, hedging through implied volatility strongly outperforms all other forms of hedging in terms of risk (and even risk/return trade off).

- Unconditional hedging as proposed by Campbell et al. (2010) does not lead to strong risk reduction, but lower expected returns. 


\section{Implied volatility as a strong hedge signal during crisis periods}

Figure 3: Correlation of global equities with six G20 currency pairs (against USD) across lagged implied FX volatility (1995:02-2015:12)
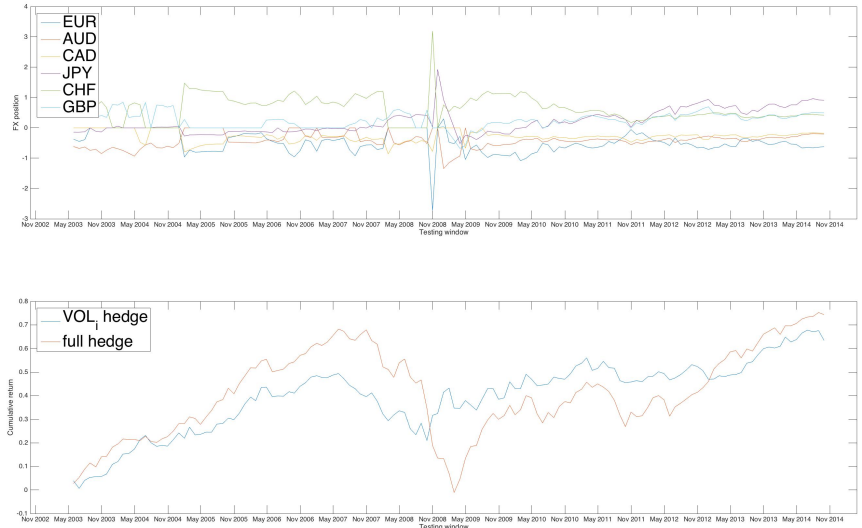


\section{Outline}

\section{(1) Contribution to literature \& Motivation}

(2) Research design

(3) Major results

(4) Conclusion 


\section{Conclusion}

- Implied FX volatility can reduce portfolio risk in and out-of sample. This is particularly true during crisis periods.

- Unconditional currency hedging as proposed by Campbell et al. (2010) does not hold up out-of-sample (higher risk than full hedging).

- Reduction in portfolio risk across the board: Variance, negative skewness, kurtosis and crash risk (drawdown) are significantly reduced.

- However, the risk reduction is no free lunch: reduction in expected returns with same or decreasing Sharpe ratio.

- So far analysis conducted with Equities and Bonds. Commodities, role of emerging market currencies and transaction costs still to be included 